\title{
3D TRACKING BASED AUGMENTED REALITY FOR CULTURAL HERITAGE DATA MANAGEMENT
}

\author{
C. Battini ${ }^{\text {a }}$, G. Landi ${ }^{\text {b }}$ \\ ${ }^{a}$ DICCA, Department of Civil, Chemical and Environmental Engineering, 16145 Genova, Via Montallegro 1, Italy - \\ carlo.battini@unige.it \\ ${ }^{\text {b }}$ ETT s.p.a., 16154 Genova Sestri Ponente, via Sestri 37, Italy - giovanni.landi@ettsolutions.com
}

\author{
Commission V, WG V/4
}

KEY WORDS: Augmented reality, Laser scanner, Tracking 3D, Reverse modelling, Survey

\begin{abstract}
:
The development of contactless documentation techniques is allowing researchers to collect high volumes of three-dimensional data in a short time but with high levels of accuracy. The digitalisation of cultural heritage opens up the possibility of using image processing and analysis, and computer graphics techniques, to preserve this heritage for future generations; augmenting it with additional information or with new possibilities for its enjoyment and use. The collection of precise datasets about cultural heritage status is crucial for its interpretation, its conservation and during the restoration processes. The application of digital-imaging solutions for various feature extraction, image data-analysis techniques, and three-dimensional reconstruction of ancient artworks, allows the creation of multidimensional models that can incorporate information coming from heterogeneous data sets, research results and historical sources. Real objects can be scanned and reconstructed virtually, with high levels of data accuracy and resolution. Real-time visualisation software and hardware is rapidly evolving and complex three-dimensional models can be interactively visualised and explored on applications developed for mobile devices.

This paper will show how a 3D reconstruction of an object, with multiple layers of information, can be stored and visualised through a mobile application that will allow interaction with a physical object for its study and analysis, using 3D Tracking based Augmented Reality techniques.
\end{abstract}

\section{INTRODUCTION}

Three-dimensional survey techniques are increasingly used today for cultural heritage studies. The collected data may be the basis for morphological and preservation analysis of objects. These survey techniques generate datasets, which are the fundamental basis on which new and general information is overlaid. This process is in a new spatial location and creates relationships between various types of results thereby giving new interpretation tools.

There are numerous 3D collection technologies available today, some of which use laser. These instruments permit the capture of complex geometries with a degree of resolution that varies depending on the technique used. The results must be defined in the design phase of the survey and must be to the scale used for visualization.

Cultural Heritage documentation often includes results rendered in scale 1:50 and, in some cases, 1:20. These scale factors are able to decipher the peculiarities of the piece very accurately, showing not only the form but also the colouring of the materials used. This data is essential for successful restoration planning and design.

By restricting the range of technologies available to scale 1:20, the use of phase-shifting approaches, with a measurement tolerance of 2-3 mm, is a good way recording morphological composition; also for statues, where this type of technology involves information discretisation and highlights the reflectivity of the material exposed to a beam of light.

Dedicated applications process this information and extract dimensional and colouring information. It is unfortunate that the data obtained is only available to those with special training in computer graphics, usually based in distant laboratories.
Other data that need to be collected during the restoration and conservation phases concern conservative and material peculiarities. This information is useful when choosing the most suitable restoration technique and materials. The complexity and the amount of collected data often leads to the creation of complex and not easily transferable maps and reports.

The project described in this article aims at making all this information available on site, simply and immediately. This system permits greater interaction with objects and delivers simple information organisation tools that also store data for easy integration with other software.

In this way, the data will always be available and will facilitate comprehension of the decay conditions.

The aim of the project is also to give basic information to unskilled users, such as tourists.

It will therefore be possible to classify the article and show precise information on the iconographic and material composition, thereby encouraging greater understanding of the design idea behind the piece of art.

\section{CASE STUDY}

This article deals with the experiment carried out on the statue of Leonardo da Vinci (Figure 1) in Piazza della Scala, Milan, sculptured by Pietro Magni, a local artist. The original planned site of the statue was in Palazzo Brera but this was changed when King Vittorio Emanuele II inaugurated the Galleria Vittorio Emanuele II in 1872, during the Second National Exhibition.

The monument consists of an octagonal base, in Pink Baveno Granite, on which four Carrara marble statues of disciples, by Giovanni Antonio Boltraffio, A Solaino, Marco d'Oggiono and 


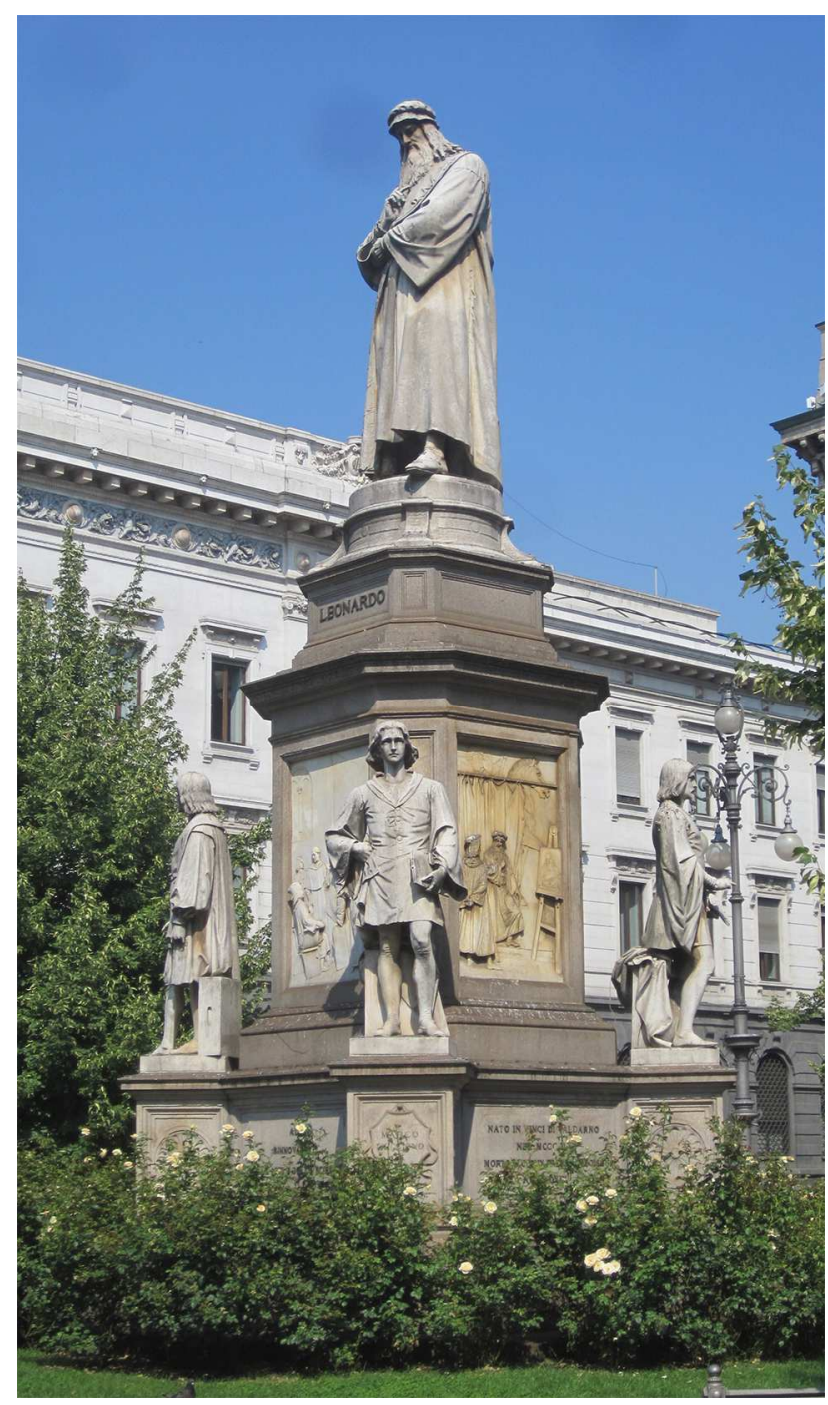

Figure 1. The statue of Leonardo da Vinci

Cesare Da Sesto, are mounted. There are also four important bas-relief works. These works show scenes of "Leonardo the painter" while painting The Last Supper, "Leonardo the sculptor" while creating the equestrian statue dedicated to Francesco Sforza, "Leonardo the architect and strategist" in the role of construction manager for the fortification works to the castles owned by the Duca Valentino di Romagna, and

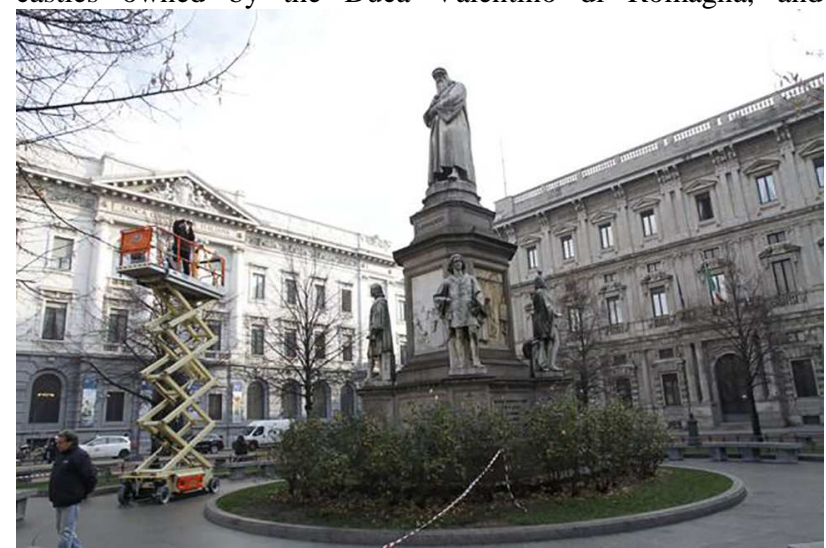

Figure 2. Steps of survey of the monument. 3D scan with elevator
"Leonardo the hydraulic engineer", struggling with the irrigation canals and waterways in Lombardy.

The austere statue of Leonardo da Vinci is mounted on the highest part of the monument.

Laser instruments were used for the geometric characterization survey of this complex monument, and an SLR camera obtained colour information. Laser measurements were made from an elevator, using a laser scanner Z+F Imager 5006h, from 24 positions and at three distinct heights, taking care to capture every detail (Figure 2). At each laser location, 12 megapixel photos were taken with a Nikon D5000 SLR camera using an 8 mm Samyang lens, mounted on a Nodal Ninja 3 with the nodal point linked to the laser's reference system. These images were then processed with PT GUI stitching software to obtain the equirectangular panoramas used to colour the individual laser scans.

During the editing process, a first union of the point cloud within the software Cyclone 8.1 (Figure 3) occurred and, at the same time,

colour information acquired by spherical panoramas was added. This resulted in an almost complete three-dimensional dataset of the statue of Leonardo, useful for the generation of orthophoto-planes and reusable in other reverse modelling software.

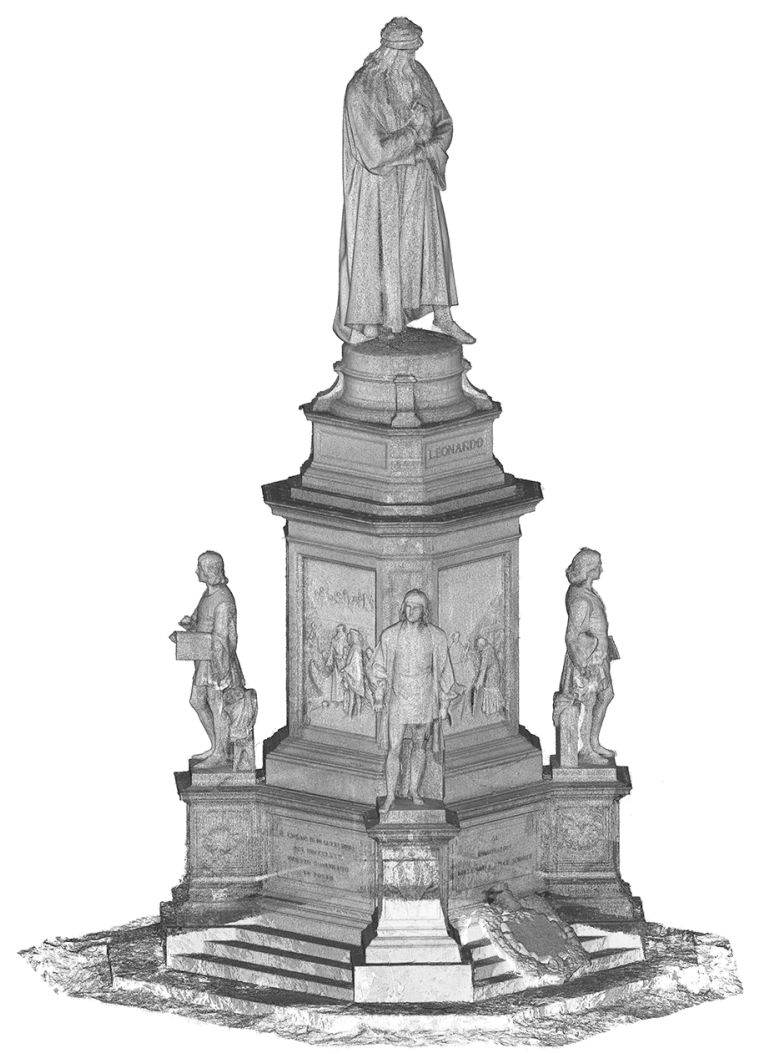

Figure 3. Point cloud of the statue registered in Cyclone

The Geomagic Studio (Figure 4) reverse modelling software was used to process the data. The single point clouds were first filtered to remove isolated points and partially acquired areas. The data were then transformed into meshes. These areas were filtered again to harmonise individual scans. After this, all the portions of the statue were aligned and merged into a single polygonal model. 


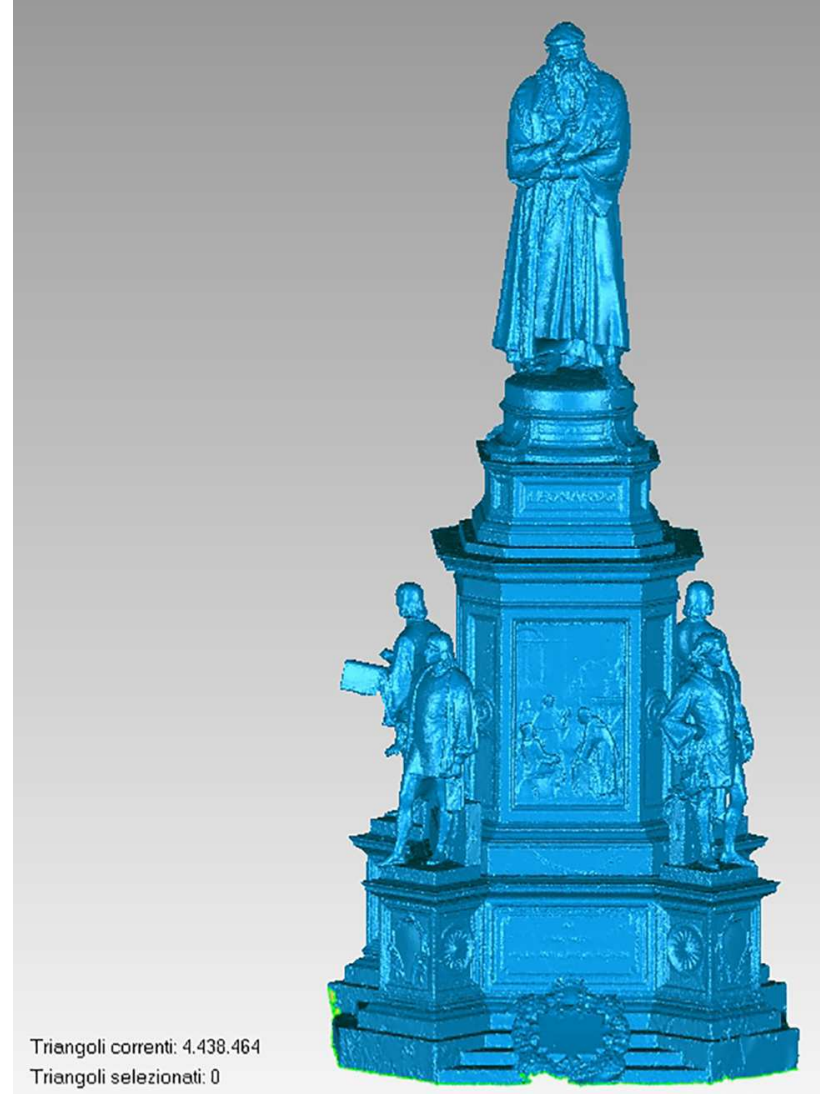

Figure 4. Reverse modelling of the monument

This software was also used to test some photographic image reprojection procedures to render the materials of the statue,finding their centres and baking the colour information onto the model's UV Maps.

One problem found was texture non-uniformity, especially in portions of the model where several pictures were taken. It was decided to edit the individual UV Maps using a photo-editing program to balance their RGB curves and make the calculated textures uniform (Figure 5).

Information on the state of conservation of the statue, fundamental for restoration planning, was also added to the geometric and colorimetric data of the monument.

Among the observed defects, it is important to note that:

the base, in Pink Baveno Granite, is marked by consistent and inconsistent deposit types, with particular reference to traces of pigeon guano deposits;

the statues of Leonardo da Vinci and the four disciples, in Carrara marble, is marked by inconsistent deposits and black crusts caused by atmospheric pollution;

in particular, the bas-reliefs placed on the four principal faces of the base are marked by inconsistent deposits and by the beginning of localized disintegration of the stone. There are also noticeable colour changes in many parts of the bas-reliefs, presumably caused by chemical alterations to the limestone surface.

This substantial information is essential for planning and implementation of subsequent phases of restoration.

\section{AUGMENTED REALITY USED FOR RESTORATION}

This project aims to be an example of the potential that Augmented Reality (AR) can offer. Architecturally, AR systems

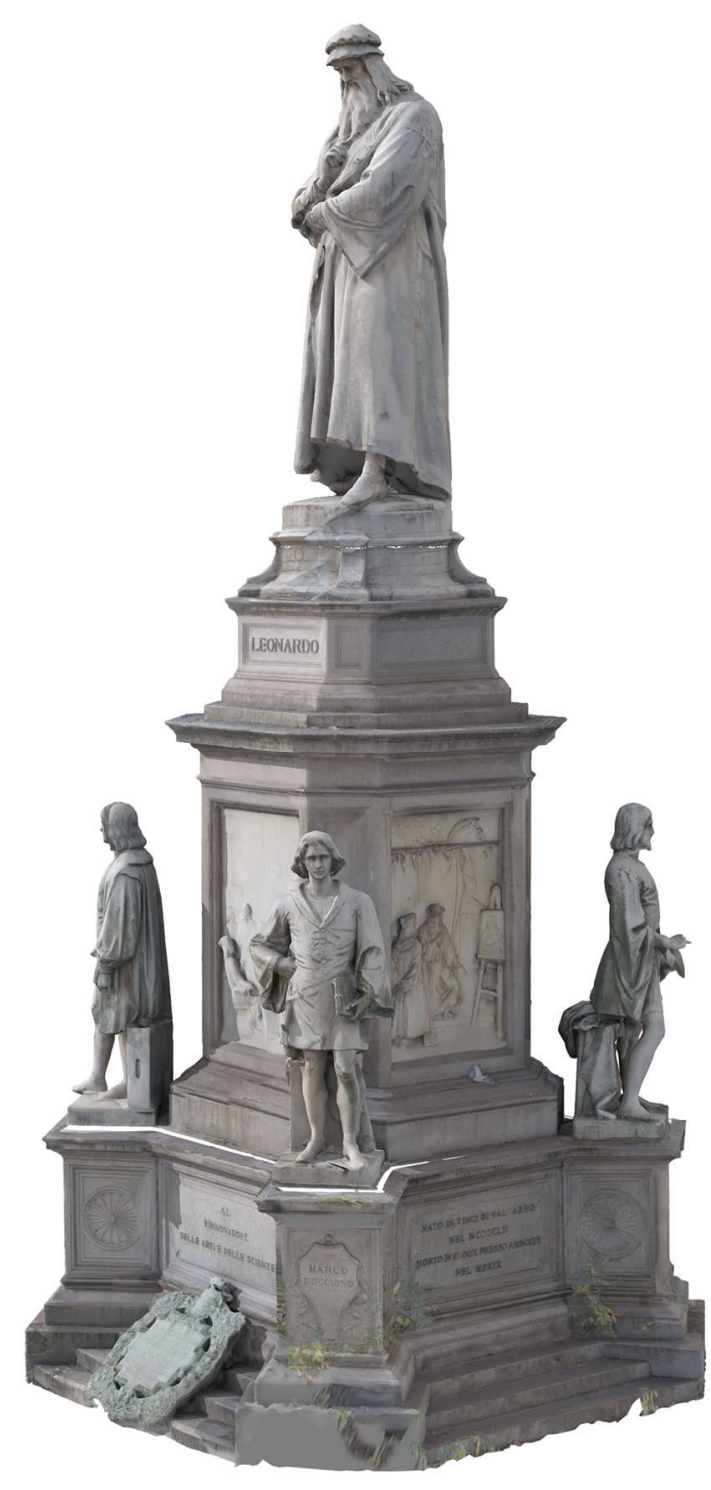

Figure 5. 3D model with applied uv map

include mechanisms to track information about user location and the position of real world objects; hardware and software to process information, and devices to show users the digital information integrated with the real environment (Azuma, 1997).

Augmented reality is usually employed using special twodimensional markers or, for special applications, using photographic imaging of the real object. Using these techniques undeniably means that $\mathrm{AR}$ cannot be used on rounded objects such as the statue of Leonardo da Vinci.

However, some studies and applications are able to implement 3D tracking, so that AR may then be used for three-dimensional objects.

The project presented in this article uses the ARmedia 3D SDK application. This tracks three-dimensional objects in space. This feature associates coherent three-dimensional information to what is being seen in the camera viewfinder. The application uploads a photoset of investigated case studies, in addition to a three-dimensional CAD model. The SDK algorithm creates relationships and correspondences between the photographs and the geometric characteristics of the model. 
With synergetic use of the edge-based tracking system function (which helps determine the position of the camera by identifying edges and corners on the three-dimensional model) and template-based tracking (a process involving off-line calculations of planar projections of the 3D model) the application creates a 3D target that can be employed to create the mobile application.

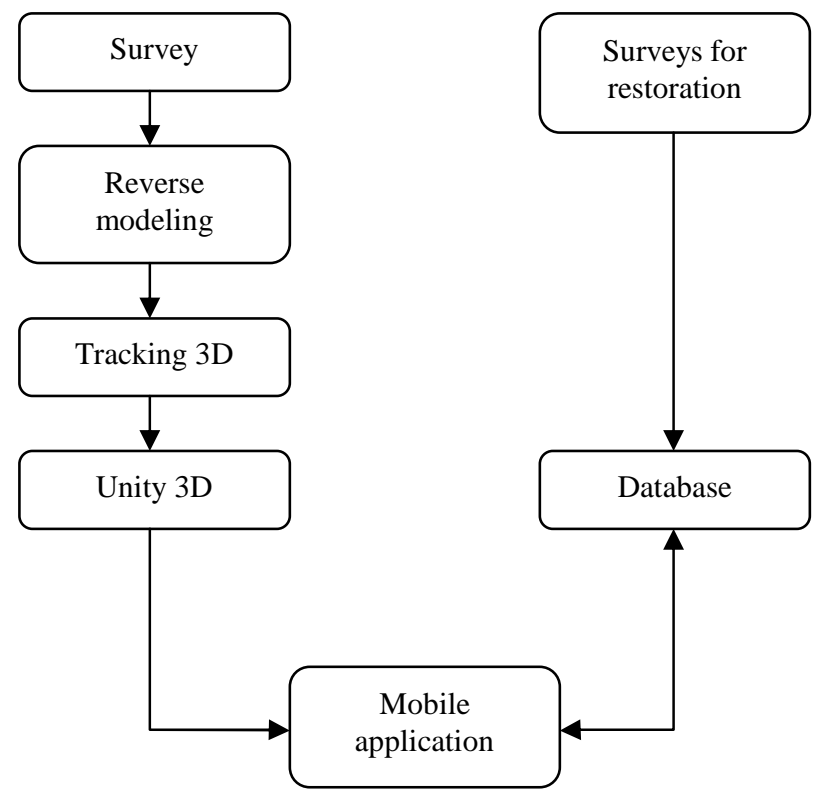

Figure 6. Scheme of the process applied to the creation of the management system

In the Leonardo da Vinci case study, a photoset of the statue was then created as well as an improved three-dimensional model for tracking calculation. This data were then entered into Unity 3D, an integrated multiplatform authoring tool that creates 3D video games and other interactive content (Figure 6). Having got a prototype AR display of Leonardo da Vinci's monument, the project focused on the preparation of the interaction utility that would be capable of handling the vast quantity of data acquired both during the survey and during the analysis of the statue's state of conservation.

The mobile unit (Figure 7) has an internal application that employs a graphic user interface (GUI), a 3D tracking system and a graphics engine for data visualization and management (Unity 3D). These components operate simultaneously, both to render the image on the device and to aid user interaction.

For the storage and sharing of data, an SQL database is necessary. This will ensure perfect integration with the project App and also with future web-based systems.

Data management script in $\mathrm{C \#}$ was then designed and written for the Unity platform. System operation buttons were assigned to each function, to be used singly or together with other buttons.

The main functions are for the insertion and editing of up-todate three-dimensional localised information, or for manual use in user-defined areas. It is therefore possible for a user to select a view of the statue, click on his device and connect to the database in order to combine a database query with spatial coordinates. The system will instantly check for previously saved information to show on the screen, or will create a new query to fill-in.
The points and sensitive areas were identified using ray cast and UV Map functions of the three-dimensional model.

Spatial coordinates of a point were identified using a function that uses ray casting along the perpendicular to the pickup point. This ray intersects the model and gives the spatial coordinates of the meeting point.

In the same way, sensitive areas are identified by multiple ray casting that define a polygonal closed in space. Theme differences in the selected area are defined by the association of distinct colours.

This association was made possible by combining RGB information to the relevant portion of the 3D model UV Map.

Other applied application tools permit point-to-point measurements. This system is not a direct part of the case study but can be very useful in positions difficult to get at from the ground, the statue of Leonardo for example.

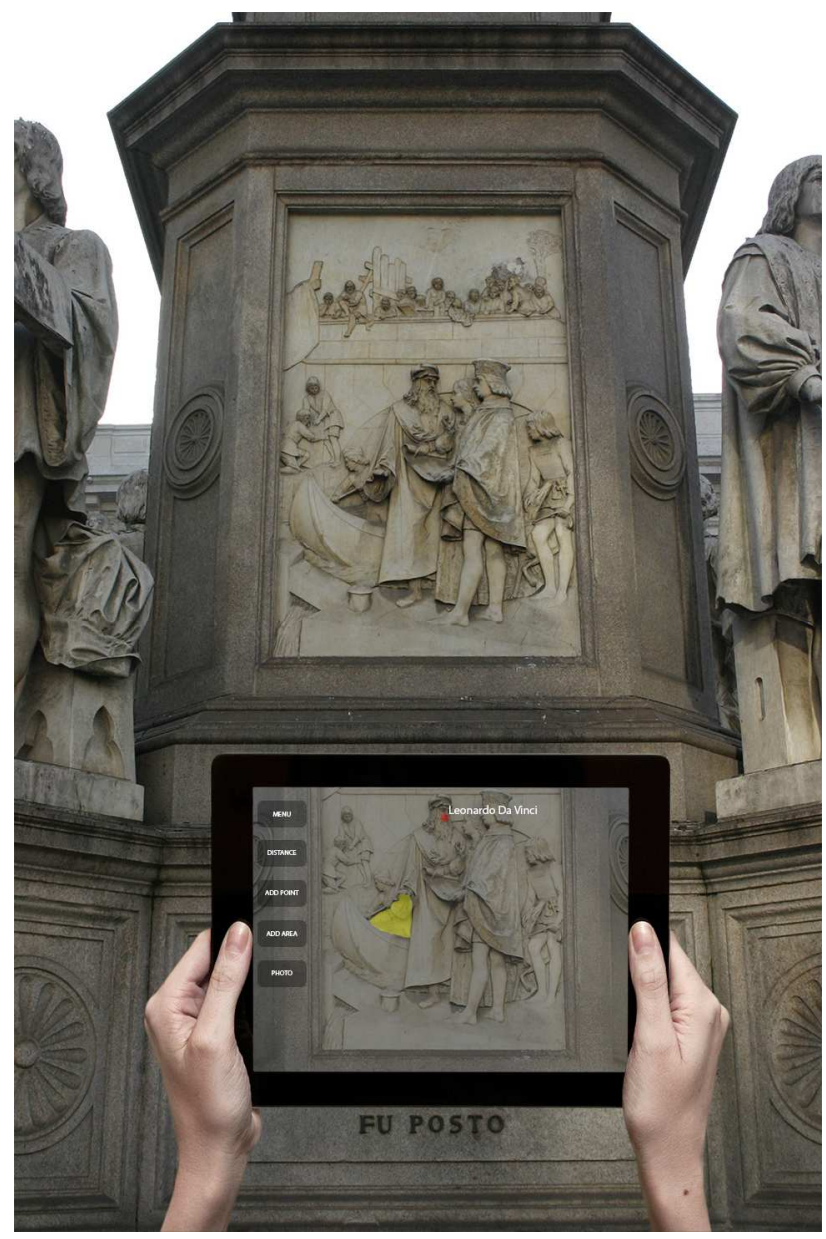

Figure 7. Use of the mobile application on the statue of Leonardo da Vinci

\section{CONCLUSIONS}

This research experimented with the use of applications based on Augmented Reality. This visual system shows great potential both for science and for tourism, and is a first step towards understanding possible innovative future applications in information management. 
Many issues still need to be resolved, mainly related to the customisation and optimisation of the graphical interface, as well as the computing engine performance.

Future research will experiment with solutions proposed in other case studies, comparing solutions and choices with restoration researchers and other possible users.

\section{ACKNOWLEDGEMENTS}

The authors would like to acknowledge and thank the developers of AR Media for the opportunity to use their application. System that can lead an important development in the field of cultural heritage.

\section{REFERENCES}

Azuma R.T., 1997. A Survey of Augmented reality. In: Presence: Teleoperators and Virtual Environments, 6, pp. 355385 .

Heckel W., Yardley J., 2004. Alexander the great: Historical sources in translation. Blackwell Publishing, Oxford, UK.

Mohammed-Amin R. K., 2010. Augmented reality: A narrative layer for historic sites. MEDes (CMD), University of Calgary.

Di Paola F., Inzerillo L., 2011. Augmented reality. The case of Salinas Museum of Palermo. In: Gambardella C. (a cura di) Le Vie dei Mercanti. S.A.V.E. Heritage, La Scuola di Pitagora Editrice, Napoli, Italy, pp. 1-7.

Battini C., 2012. Rilievo digitale e restituzione, concetti base ed esempi. Alinea editrice, Firenze, Italy, pp. 114.

Battini C., 2014. Augmented reality and cultural heritage. New systems of representation. In: V Congreso Internacional de Expresión Gráfica en Ingeniería, Arquitectura y Carreras Afines y XI Congreso Nacional de Profesores de Expresión Gráfica en Ingeniería, Arquitectura y Carreras Afines, Rosario, Argentina, pp. 452-457.

Bonacini E., 2014. La realtà aumentata e le app culturali in Italia: storie da un matrimonio in mobilità, in: Il Capitale Culturale. Studies on the Value of Cultural Heritage, IX, EUM Edizioni Università di Macerata, Italy, pp. 89-121.

Landi G., 2014. AR for the comprehension of linear perspective in the Renaissance masterpiece The Holy Trinity (Masaccio, 1426). In: Mixed and Augmented Reality - Media, Art, Social Science, Humanities and Design (IMSAR-MASH'D), 2014 IEEE International Symposium on, Munich, German, pp. 69-70. 